\title{
https://doi.org/10.46813/2021-134-043 \\ INHOMOGENEOUS TRAVELLING-WAVE ACCELERATING SECTIONS AND WKB APPROACH
}

\section{M.I. Ayzatsky \\ National Science Center “Kharkov Institute of Physics and Technology”, Kharkiv, Ukraine E-mail: aizatsky@kipt.kharkov.ua}

The paper presents the results of a study of the possibility of using the WKB approach to describe Inhomogeneous Travelling-Wave Accelerating Sections. This possibility not only simplifies the calculation, but also allows the use of simpler physical models of transient processes. Using the traveling wave concept simplifies the understanding of pulsed-excited ITWAS transients and the development of methods to mitigate their effect on beam parameters.

PACS: 29.20.- c; 84.40.Az

\section{INTRODUCTION}

To explore possibility of using difference equations and approximate methods, we have proposed a simple but rigorous model of Inhomogeneous Travelling-Wave Accelerating Sections (ITWAS) [1]. This model is based on the method of Coupled Integral Equations (CIE) (see, for example, [2]). Using the theory of solving matrix equations (see [3, 4] and the literature cited there) and the decomposition method [5], we obtained new matrix difference equations, on the basis of which various approximate approaches, including the WKB approach, can be developed. In this paper we present the results of using proposed approach to study the properties of different accelerating sections.

\section{MODEL OF ITWAS. BASIC EQUATIONS}

In this section, we present the basic equations of the model, the derivation of which is presented in the work [1]. We consider the chain of $N_{R}$ cylindrical resonators that couple through cylindrical openings in the thin diaphragms. End resonators through cylindrical openings are connected to the cylindrical waveguides. The resonator volumes are filled with a dielectric, the dielectric constant of which has an imaginary part $\varepsilon=1+i \varepsilon^{\prime \prime}$. With this choice one can take into account the losses and preserve the orthogonality of the waveguide cylindrical functions.

Electric field in the $k$-th resonator is determined as

$$
\begin{gathered}
E_{z}^{(k)}\left(z_{k}, r=0\right)= \\
\left(T_{1}^{E(k)}\left(z_{k}\right)\right)^{T} C^{(k)}-\left(T_{2}^{E(k)}\left(z_{k}\right)\right)^{T} C^{(k+1)},
\end{gathered}
$$

where $0<z_{k}<d_{k}, T_{1}^{E(k)}\left(z_{k}\right), T_{2}^{E(k)}\left(z_{k}\right), C^{(k)}$ are the complex $N_{m}$-dimensional vectors (see their definition in [1]). Vector $C^{(k)}$ determines the electric field on the opening of the $k$-th diaphragm

$$
E_{r}^{(k)}=\sum_{s=1}^{N_{m}} C_{s}^{(k)} \varphi_{s}\left(\frac{r}{a_{k}}\right),
$$

where $\varphi_{s}$ is a set of basis functions. We used the complete set of functions that fulfil the edge condition on the diaphragm rims as the basis functions (the Meixner basis). We use such Meixner basis [6]

$$
\varphi_{s}(r)=2 \sqrt{\pi} \frac{\Gamma(s+1)}{\Gamma(s-0.5)} \frac{1}{\sqrt{1-r^{2}}} P_{2 s-1}^{-1}\left(\sqrt{1-r^{2}}\right),
$$

where $P_{n}^{m}(x)$ are Legendre functions (or spherical functions) of the first kind [7].

Making special decomposition ${ }^{1}[1,5]$

$$
C^{(k)}=\Xi^{(k)}\left(\tilde{C}^{(k, 1)}+\tilde{C}^{(k, 2)}\right),
$$

we get the system of difference matrix equations:

$$
\begin{aligned}
& \tilde{C}^{(k+1,1)}=\left\{M^{(k+1,1)}+\tilde{M}^{(k+1,1)}\left(M^{(k, 1)}-M^{(k+1,1)}\right)\right\} \tilde{C}^{(k, 1)}+ \\
& +\tilde{M}^{(k+1,1)}\left(M^{(k, 2)}-M^{(k+1,2)}\right) \tilde{C}^{(k, 2)}, \\
& \tilde{C}^{(k+1,2)}=\left\{M^{(k+1,2)}+\tilde{M}^{(k+1,2)}\left(M^{(k, 2)}-M^{(k+1,2)}\right)\right\} \tilde{C}^{(k, 2)}+ \\
& +\tilde{M}^{(k+1,2)}\left(M^{(k, 1)}-M^{(k+1,1)}\right) \tilde{C}^{(k, 1)},
\end{aligned}
$$

where $k=2,3, \ldots, N_{R}-2, M$ are complex $N_{m} \times N_{m}$ matrices (see their definition in [1]).

If elements of matrices $M^{(k, i)}$ vary sufficiently slowly with $k$, then the differences $\left|M_{s, m}^{(k+1, i)}-M_{s, m}^{(k, i)}\right|$ are the small values and we can neglect some of them and get:

Eikonal approximation

$$
\begin{aligned}
& \tilde{C}^{(k+1,1)}=M^{(k, 1)} \tilde{C}^{(k, 1)}, \\
& \tilde{C}^{(k+1,2)}=M^{(k, 2)} \tilde{C}^{(k, 2)},
\end{aligned}
$$

WKB approximation

$$
\begin{aligned}
& \left.\tilde{C}^{(k+1,1)}=\tilde{\tilde{M}}^{(k+1,1)}{\underset{\sim}{(k, 1)}}^{(k+1)}\left(M^{(k, 1)}-M^{(k+1,1)}\right)\right\} \tilde{C}^{(k, 1)}, \\
& =\left\{M^{(k+1,1)}+\tilde{M}^{(k+1,1)}\left(\tilde{\tilde{M}}^{(k+1,2)} \tilde{C}^{(k, 2)}=\right.\right. \\
& \tilde{C}^{(k+1,2)}= \\
& =\left\{M^{(k+1,2)}+\tilde{M}^{(k+1,2)}\left(M^{(k, 2)}-M^{(k+1,2)}\right)\right\} \tilde{C}^{(k, 2)} .
\end{aligned}
$$

Electric field in the $k$-th resonator can be divided into "forward" and "backward" parts:

$$
\begin{gathered}
E_{z}^{(k)}=E_{z}^{(k, 1)}+E_{z}^{(k, 2)}= \\
=\left[\left(T_{1}^{E(k)}\right)^{T} \Xi^{(k)} \tilde{C}^{(k, 1)}-\left(T_{2}^{E(k+1)}\right)^{T} \Xi^{(k+1)} \tilde{C}^{(k+1,1)}\right]+ \\
+\left[\left(T_{1}^{E(k)}\right)^{T} \Xi^{(k)} \tilde{C}^{(k, 2)}-\left(T_{2}^{E(k+1)}\right)^{T} \Xi^{(k+1)} \tilde{C}^{(k+1,2)}\right] .
\end{gathered}
$$

In the case of homogeneous waveguide $\left(C^{(k, 2)}=0\right.$, travelling wave regime)

${ }^{1}$ This decomposition can be considered as generalized decomposition into "forward" and "backward" solutions. 


$$
\begin{gathered}
E_{z}^{(k)}(z+d k, r=0)= \\
=\Omega^{T}(z)\left(M^{(1)}\right)^{k} C^{(0,1)}=\Omega^{T} U \Lambda^{(1) k} U^{-1} C^{(0,1)},
\end{gathered}
$$

where $0<z<d, \Omega=T_{1}^{E}(z)-M^{(1)} T_{2}^{E}(z), \quad U$ is the matrix of eigen vectors, $\Lambda^{(1)}=\operatorname{diag}\left(\lambda_{1}^{(1)}, \lambda_{2}^{(1)}, \ldots, \lambda_{N_{m}}^{(1)}\right)$ and $\lambda_{s}^{(1)}$ are the solution of the characteristic equation of homogeneous waveguide (see [1]).

From (9) it follows that we take into account $N_{m}$ eigen waves, including the evanescent ones. Indeed, if $C^{(0,1)}$ is a superposition of $N_{m}$ eigen modes $C^{(0,1)}=\sum_{s=1}^{N_{m}} B_{s} U_{s}$, we have

$$
E_{z}^{(k)}(z+k d, r=0)=\Omega^{T} \sum_{s=1}^{N_{m}} B_{s} \lambda_{s}^{(1) k} U_{s}
$$

\section{SMOOTH TRANSITION BETWEEN TWO DISK LOADED WAVEGUIDES}

To demonstrate the correctness and capabilities of the proposed rigorous model and approximate approaches, let us consider the classical problem of connecting two homogeneous waveguides using a smooth transition between them.

Consider the chain of resonators in which the aperture radii and resonator radii vary as

$$
\begin{aligned}
& a_{k}=\frac{a_{I}+a_{I I}}{2}+\frac{a_{I}-a_{I I}}{2} \frac{\operatorname{arctg}\left\{\alpha\left(k-k_{0}\right)\right\}}{\operatorname{arctg}\left\{\alpha\left(3-k_{0}\right)\right\}}, \\
& k=3, \ldots, N_{R}-1, \\
& b_{k}=\frac{b_{I}+b_{I I}}{2}+\frac{b_{I}-b_{I I}}{2} \frac{\operatorname{arctg}\left\{\alpha\left(k_{0}-k\right)\right\}}{\operatorname{arctg}\left\{\alpha\left(k_{0}-3\right)\right\}}, \\
& k=3, \ldots, N_{R}-2,
\end{aligned}
$$

where $N_{R}=2 k_{0}-1=201, a_{I}=0.99 \mathrm{~cm}, b_{I}=4.08896 \mathrm{~cm}$ $\left(\beta_{g, I}=v_{g, I} / c=0.022\right) \quad$ and $\quad a_{I I}=0.65 \mathrm{~cm}$, $b_{I I}=4.03934 \mathrm{~cm}\left(\beta_{g, I I}=v_{g, I I} / c=0.0065\right)$ are the aperture radii and resonator radii of two homogeneous diskloaded waveguides. At frequency $f=2.856 \mathrm{GHz}$ these waveguides have phase shift per cell $\varphi_{0}=2 \pi / 3$. All resonators have the same length $d=3.4989 \mathrm{~cm}$. The sizes of couplers $\left(a_{1}, b_{1}\right.$ and $\left.a_{N_{R}+1}, b_{N_{R}}\right)$ were chosen from the condition of matching homogeneous diskloaded waveguides with cylindrical waveguide with radius $b_{w}=4.2 \mathrm{~cm}$.

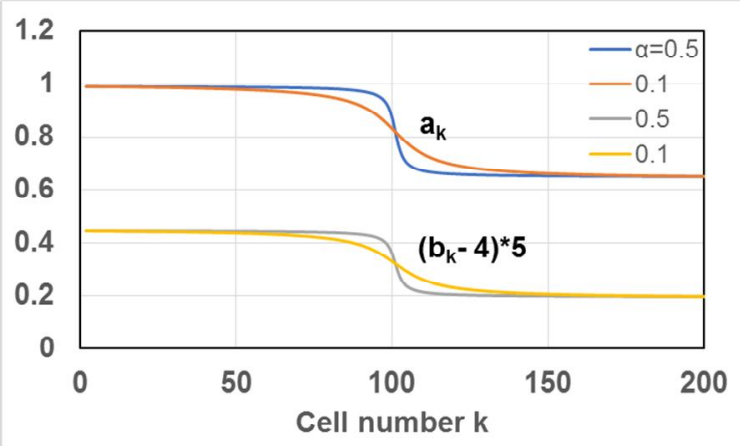

Fig. 1. The dependences of the aperture radii and resonator radii on the cell number

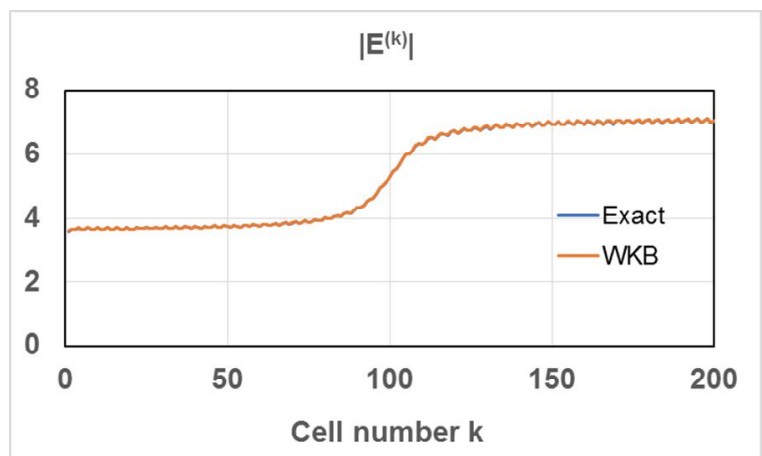

Fig. 2. Electric field distribution for smooth transition

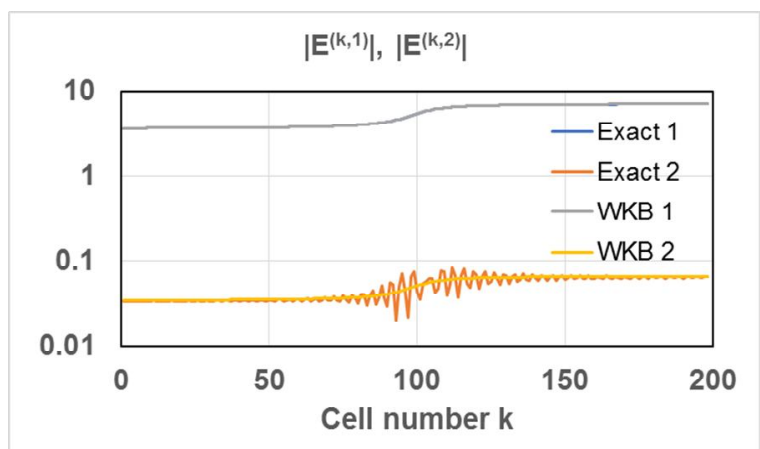

Fig. 2. The "forward" and "backward" parts of electric field distribution for smooth transition

In this section we will consider the lossless case $\varepsilon^{\prime \prime}=0$. The dependences of the aperture radii and resonator radii for the considered two values of parameter $\alpha$ are presented in Fig. 1.

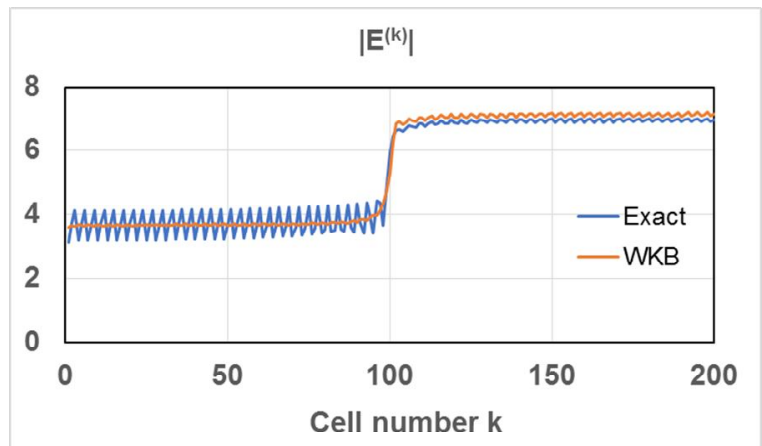

Fig. 3. Electric field distribution for a steeper transition

For smooth transition $(\alpha=0.1)$ the exact and approximate $(\mathrm{WKB})$ solutions practically coincide (see Fig. 2, $\left.\mathrm{E}^{(k)}=E_{z}^{(k)}(d / 2+d k, r=0)\right)$.

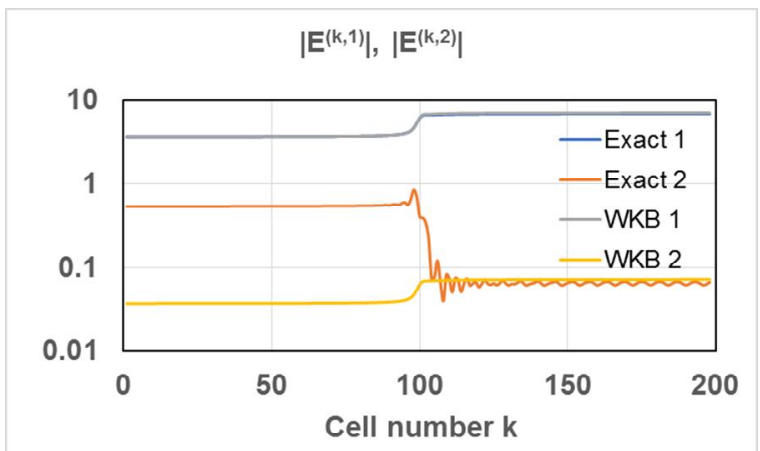

Fig. 4. The "forward" and "backward" parts of electric field distribution for a steeper transition

The same is true for "forward" and "backward" parts of electric field (Fig. 3). The detailed analysis shows 
that they are indeed "forward" and "backward", as their phases change in different directions.

For a steeper transition $(\alpha=0.5)$ there are difference between the exact and WKB solutions (Fig. 4) as a reflected wave arose in the region before transition (Fig. 5).

The presented above results show that the WKB model correctly describe the considered cavity chain with a slow change in the parameters.

\section{INHOMOGENEOUS TRAVELLING- WAVE ACCELERATING SECTIONS}

The ITWASs have a unique property. Nobody knows the geometric sizes of resonators in the real section with sufficient accuracy ${ }^{2}$. Only the sizes of coupling holes have definite values. There are several reasons for it. The first reason is associated with the difficulty to conduct numerical modelling with an accuracy of fractions of a micrometer. The second - take into account all brazing peculiarities. But the main reason is that their knowledge does not give us useful information about the distribution of the main ITWAS characteristic - the electric field. Therefore, after preliminary selection of the resonator dimensions, fabrication and brazing, a tuning procedure is used to obtain the required electric field distribution. For today, there are two most using tuning methods: phase Ph-method and S-method. In the first method the phase shifts between resonators are tuned to the desired values by slight changing of the cavity radii (see, for example, [8]). In the second method the combinations of field meanings in some points of several cells are reduced to the desired values by the same actions [9]. For the ITWASs with phase shift $\varphi=2 \pi / 3$ the tuning condition has a form

$$
\left|\operatorname{Re}\left(S^{(k)}\right)\right| \Rightarrow \min ,
$$

where

$$
S^{(k)}=-\frac{\mathrm{E}^{(k-1)}+\mathrm{E}^{(k)}+\mathrm{E}^{(k+1)}}{\sqrt{3} \mathrm{E}^{(k)}} .
$$

For tuned homogeneous waveguide $S^{(k)}$ have zero real parts

$$
S^{(k)}=i \alpha d,
$$

where $\alpha$ is an attenuation coefficient.

For more details of the $\mathrm{S}$ method and its restriction, see $[10,11]$.

The ITWASs can be divided into three groups: with weakly $[12,13,14]$, medium $[1,15]$ and strong $[16,17]$ inhomogeneity. There are sections with very fast changes (sections with a quasi-constant gradient) [18, 19]. In this work we shall consider the properties of sections with medium and strong inhomogeneity.

First, we consider the properties of waveguides that can be base for the constant gradient section. It is known that for constant electric field strength the RF power must change linearly with distance [8]. A law of variation of the aperture radii was chosen so that the group velocity linearly drops from $\beta_{g, I}=0.022$ to

${ }^{2}$ Excluding sections that were assembled using diffusion bonding, requiring no tuning in the case of correct choosing of resonator frequencies.

ISSN 1562-6016. BAHT. 2021. № 4(134) $\beta_{g, I I}=0.0065$ along a chain of 81 resonators. The selected values of group velocity are similar to those in the SLAC section [8].

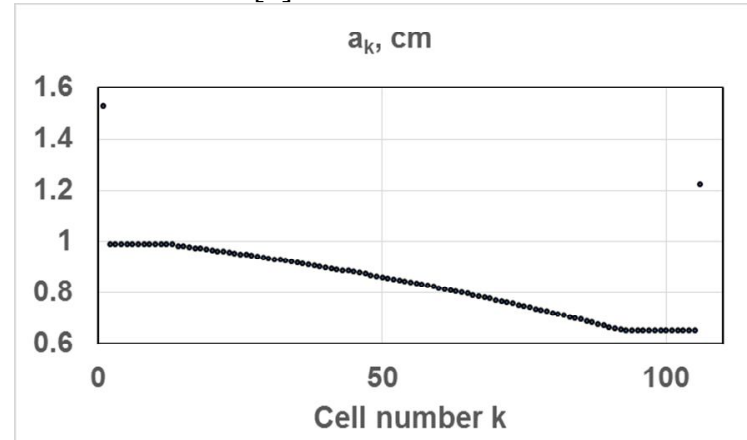

Fig. 5. The dependence of the aperture on the cell number for the constant gradient section

Earlier, the results of the study of tuning methods [11] were obtained without taking into account losses of the RF field. We introduce losses by filling the resonators with media which permittivity is complex $\varepsilon=1+i \varepsilon^{\prime \prime}$. The value of losses $\left(\varepsilon^{\prime \prime}=10^{-4}\right)$ was chosen from the condition of constant amplitude of the axial electric field at the resonator centers. To eliminate the influence of couplings on the calculation results, we placed 10 identical resonators before and 10 after the inhomogeneous chain (Fig. 6).

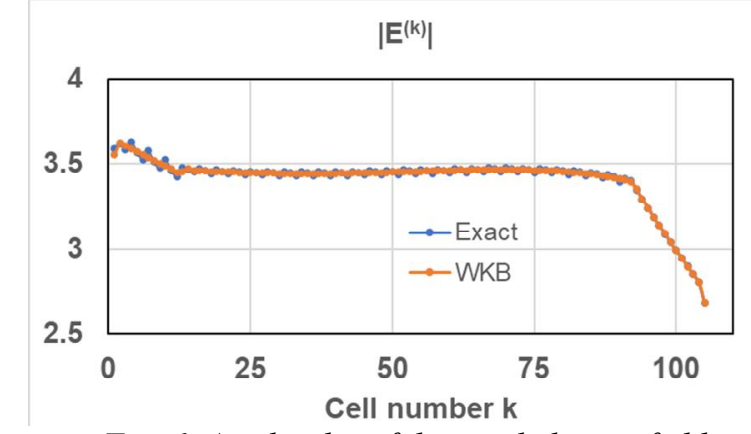

Fig. 6. Amplitudes of the axial electric field in the middle of resonators ( $S$-method)

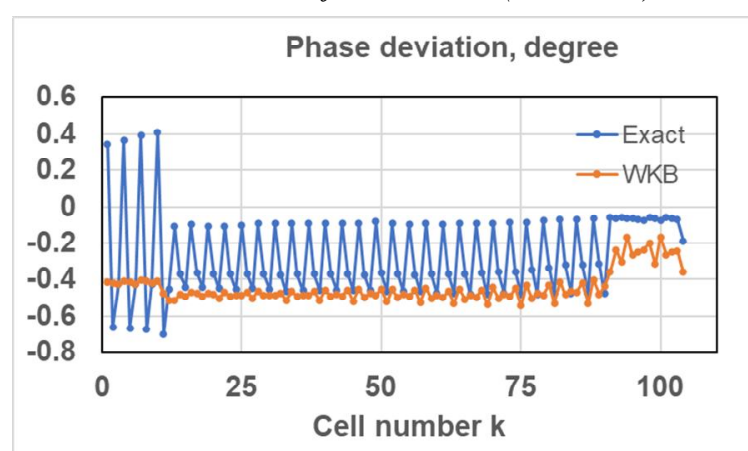

Fig. 7. Phase deviation in the middle of resonators from the $2 \pi k / 3$ law (S-method)

Characteristics of axial electric field distributions (we remind that $\mathrm{E}^{(k)}=E_{z}^{(k)}(d / 2+d k, r=0)$ ) after tuning by S-method are presented in Fig. 7, 8. Tuning process was started from the end cells. 


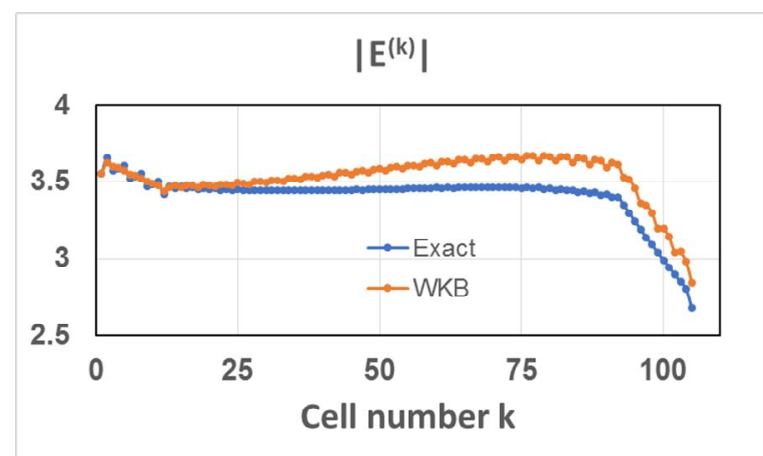

Fig. 8. Amplitudes of the axial electric field in the middle of resonators (Ph-method)

From these results it follows that the WKB approach correctly (except for small phase deviations) describe an IT WAS that is similar to the SLAC section.

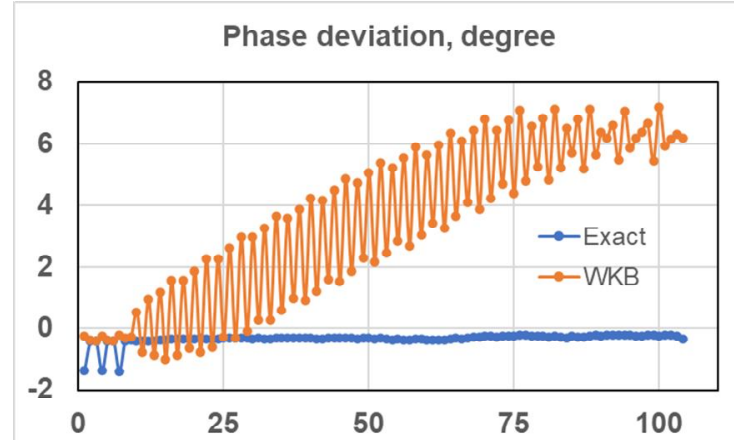

Fig. 9. Phase deviation in the middle of resonators from the $2 \pi k / 3$ law (Ph-method)

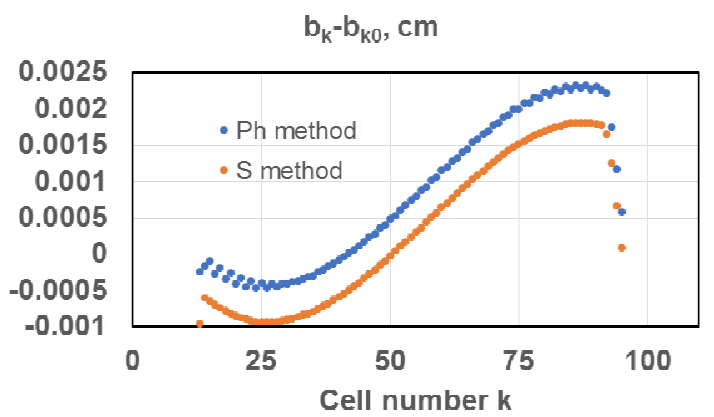

Fig. 10. Deviation of resonator radii from some smooth distribution $b_{k, 0}$ as a function of cell number. For clarity, the brown curve has been shifted down by $5 \mu \mathrm{m}$

When using $\mathrm{Ph}$ tuning methods, we get the same amplitude distribution (Fig. 9) and slightly better phases (Fig. 10). However, WKB approach cannot be used in this case (see Figs. 9, 10), as it diverges. This divergence is not related to the presence of a turning point. The reason for the divergence of the WKB method is the nonsmoothed distribution of the resonator radii when using a phase tuning. Indeed, from Fig. 11 it follows that there are small but fast oscillations of the resonator radii.

It is interesting to note that if $\operatorname{Re}\left(S^{(k)}\right)$ change significantly when tuning (see Fig. 12), in $\operatorname{Im}\left(S^{(k)}\right)$ virtually no change occurs (see Fig. 13). This parameter has no physical meaning in nonuniform waveguides, since it follows from Fig. 13 that it can even be negative (compare with (15)).

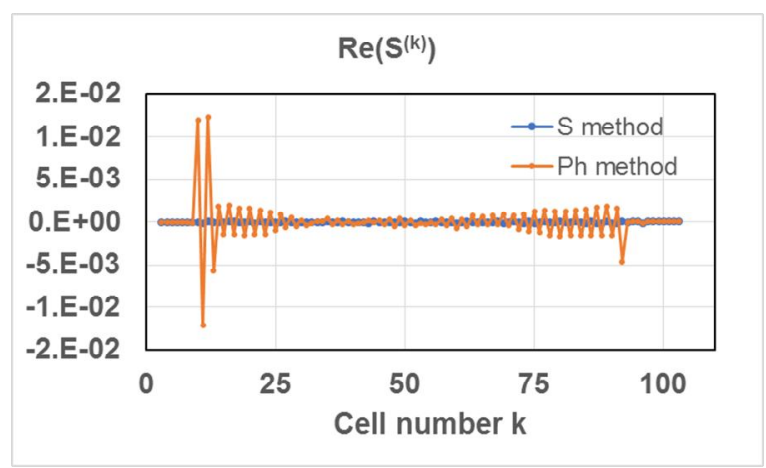

Fig. 11. $\operatorname{Re}\left(S^{(k)}\right)$ after tuning

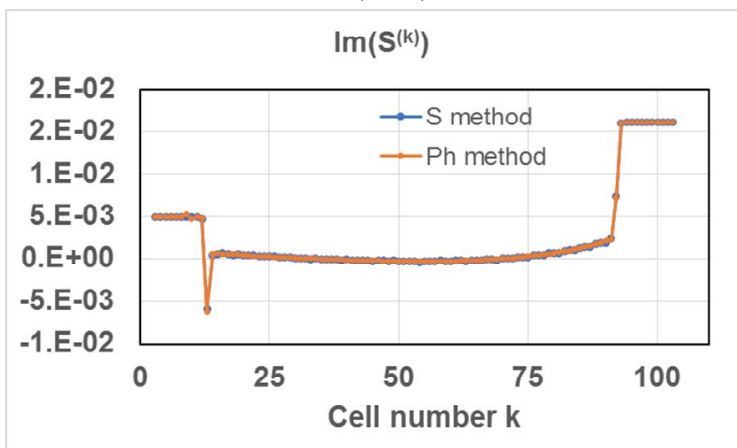

Fig. 12. $\operatorname{Im}\left(S^{(k)}\right)$ after tuning

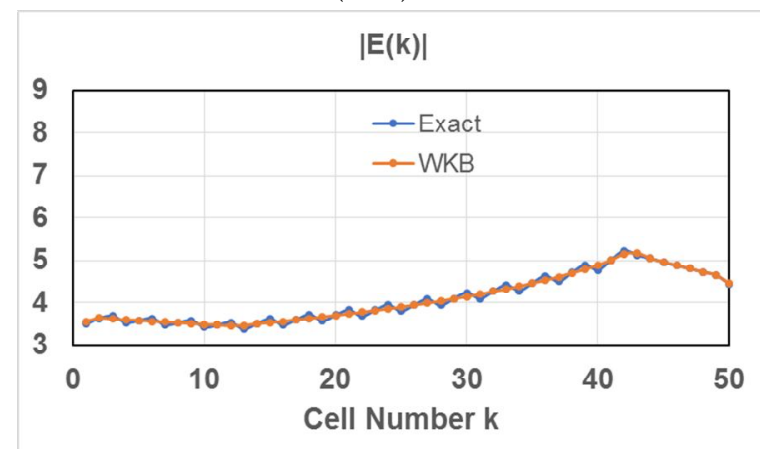

Fig. 13. Amplitudes of the axial electric field in the middle of resonators (S method)

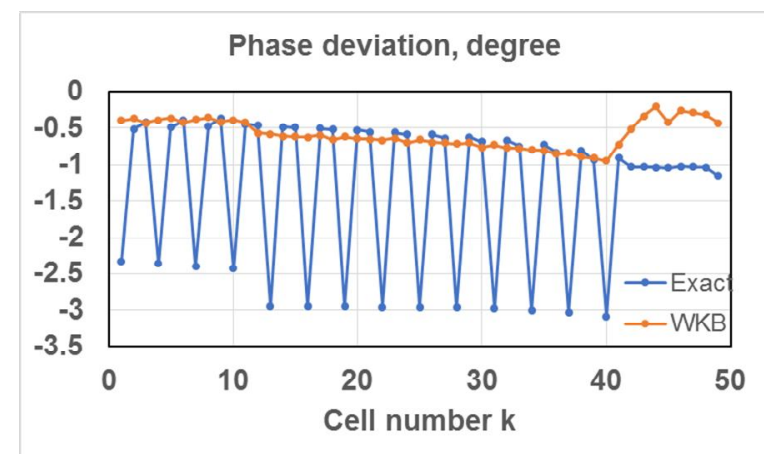

Fig. 14. Phase deviation in the middle of resonators from the $2 \pi k / 3$ law (S method)

Let's reduce the number of non-uniform cells in the chain from 81 to 31 with the same sizes of the end cells. For such waveguide $\mathrm{S}$ and $\mathrm{Ph}$ tuning methods give the same amplitude distributions (compare Figs. 14 and 16) and different phase distributions (compare Figs. 15 and 17). Ph method gives smaller phase deviations. 


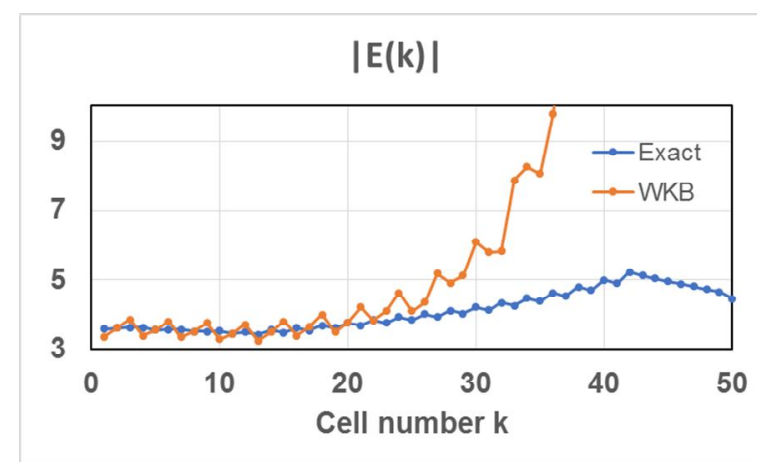

Fig. 15. Amplitudes of the axial electric field in the middle of resonators (Ph-method)

Phase deviation, degree

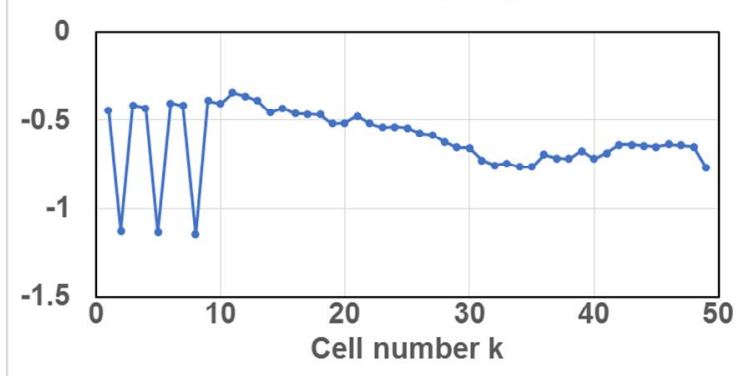

Fig. 16. Phase deviation in the middle of resonators from the $2 \pi k / 3$ law (Ph-method)

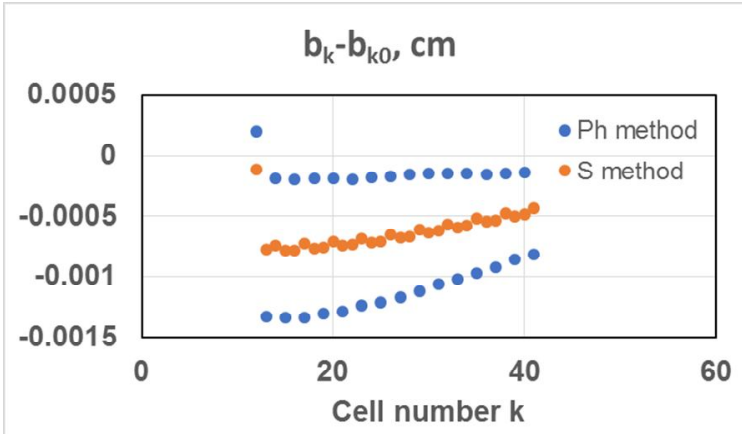

Fig. 17. Deviation of resonator radii from some smooth distribution $b_{k, 0}$

Using the WKB approach for describing such section become problematic - for the $\mathrm{S}$ tuning method there is discrepancy in phase distributions, for the $\mathrm{Ph}$ tuning method the WKB method is diverges. In this case the divergence is much stronger because the cell radii undergo larger oscillations (Fig. 18)

\section{CONCLUSIONS}

What does the divergence of the WKB method indicate? It indicates that the transients in this case will differ from those that arise in waveguides with smooth changes (a forward wave with a front moving with the group velocity). There will be multiple reflections from irregularities. For SLAC-type sections the oscillations of the resonator radii are small and the effect of multiple reflections on the transients will also be small. However, for short sections with strong inhomogeneity multiple reflections can significantly change the transients.

To avoid this, it is recommended to use tuning methods that give the required amplitude and phase distributions together with a smooth variation of the resonator frequencies. This is not an easy problem. To date, there is no tuning technique that could guarantee such a distribution of resonator frequencies.

\section{REFERENCES}

1. M.I. Ayzatsky. Modelling of Inhomogeneous DiskLoaded Waveguides: Matrix Difference Equations and WKB Approximation https://arxiv.org/abs/ 2010.10349, 2020.

2. S. Amari, J. Bornemann, and R. Vahldieck. Accurate analysis of scattering from multiple waveguide discontinuities using the coupled integral equation technique // J. Electromag. Waves Applicat. 1996, v. 10, p. 1623-1644.

3. Roger A. Horn, Charles R. Johnson. Topics in matrix analysis. Cambridge University Press, 1994.

4. Matteo Cepale, Renato Spigler. Liouville-Green (WKB) asymptotics for almost-diagonal linear second-order matrix difference equations, Journal of Difference Equations and Applications. 2017, v. 23, № 10, p. 1707-1736.

5. M.I. Ayzatsky. Transformation of the linear difference equation into a system of the first order difference equations https://arxiv.org/abs/1806.04378, 2018.

6. S.I. Eminov. Meixner basis in the disk diffraction problem // ZhTF. 2007, v. 77, № 3, p. 96-99.

7. I.S. Gradshteyn, I.M. Ryzhik. Table of Integrals, Series, and Products, Seventh Edition. AP, 2007, p. 974.

8. R.B Neal, General Editor. The Stanford Two-Mile Accelerator. New York, W.A. Benjamin, 1968.

9. T. Khabiboulline, V. Puntus, M. Dohlus, et al. A new tuning method for traveling wave structures. Proceedings of PAC95, p. 1666-1668; T. Khabiboulline, M. Dohlus, N. Holtkamp. Tuning of a 50-cell costant gradient S-band travelling wave accelerating structure by using a nonresonant perturbation method. Internal Report DESY M-95-02, 1995.

10. M.I. Ayzatsky, V.V. Mytrochenko. Electromagnetic fields in nonuniform disk-loaded waveguides. Problems of Atomic Science and Technology. 2016, № 3, p.3-10; M.I. Ayzatsky, V.V. Mytrochenko. Electromagnetic fields in nonuniform disk-loaded waveguides. https://arxiv.org/abs/1503.05006, 2015.

11. M.I. Ayzatsky, V.V. Mytrochenko. Numerical design of nonuniform disk-loaded waveguides. https://arxiv.org/abs/1604.05511, 2016; M.I. Ayzatsky, V.V. Mytrochenko Numerical investigation of tuning method for nonuniform disk-loaded waveguides. https://arxiv.org/abs/1606.04292, 2016.

12. W. Bertozzi, J. Haimson, C.P. Sargent, et al. MIT high duty, high intensity $400 \mathrm{MeV}$ accelerator // Proceedings of the PAC1967, p. 191-195.

13. H. Leboutet, G. Azam, F. Netter, et al. First operatiopn of the high duty cycle Saclay electron linac (A.L.S), Proceedings of the PAC1969, p. 299-303.

14. M. Schmitz. The S-band linear collider test facility at DESY // Proceedings of the PAC1997, p. 569-571

15. T. Tomimasu. A $500 \mathrm{MeV}$ Low Operating Cost Electron Linac // Proceedings of the PAC1981, p. 3523-3525. 
16. M.I. Ayzatsky, E.Z. Biller. Development of Inhomogeneous Disk-Loaded Accelerating Waveguides and RF-coupling // Proceedings of Linac96, 1996, v. 1, p. 119-121.

17. E. Jensen. CTF3 drive beam accelerating structures. Proceedings of Linac2002. 2002, p. 34-36

18. G. Bienvenu, J.C. Bourdon, P. Brunet, et al. Accelerating Structure Developments for the LEP Injector
Linacs (LIL). Proceedings of LINAC1984, 1984, p. 463-465.

19. E.Z. Biller, A.N. Dovbnya, V.A. Vishnyakov. New Accelerating Sections for the $2 \mathrm{GeV}$ Linac // Proceedings of PAC1989. 1989, p. 2021-2024.

Article received 23.04.2021

\section{НЕОДНОРОДНЫЕ УСКОРЯЮЩИЕ СЕКЦИИ НА БЕГУЩЕЙ ВОЛНЕ И ВКБ-ПРИБЛИЖЕНИЕ}

\section{Н.И. Айзацкий}

Представлены результаты исследования возможности использования ВКБ-подхода для описания неоднородных ускоряющих секций на бегущей волне. Эта возможность не только упрощает расчет, но и позволяет использовать более простые физические модели переходных процессов. Использование концепции бегущей волны упрощает понимание переходных процессов в секциях с импульсным возбуждением и разработку методов уменьшения их влияния на параметры пучка.

\section{НЕОДНОРІДНІ ПРИСКОРЮЮЧІ СЕКЦІЇ НА ХВИЛІ, ЩО БІЖИТЬ, І ВКБ-НАБЛИЖЕННЯ}

\section{M.I. Айзацький}

Представлено результати дослідження можливості використання ВКБ-підходу для опису неоднорідних прискорюючих секцій на хвилі, що біжить. Ця можливість не тільки спрощує розрахунок, а й дозволяє використовувати більш прості фізичні моделі перехідних процесів. Використання концепції біжної хвилі спрощує розуміння перехідних процесів у секціях з імпульсним збудженням і розробку методів зменшення їх впливу на параметри пучка. 